\title{
Review: the risk of neonatal mortality is increased in pregnant women who continue to use heroin while receiving methadone
}

\author{
Hulse GK, Milne E, English DR, et al. Assessing the relationship between maternal opiate use and neonatal mortality. Addiction \\ 1998 Jul;93:1033-42.
}

\section{Question}

Is there an association between maternal opiate use and neonatal mortality?

\section{Data sources}

English language studies were identified by searching Medline (1966 to June 1996) using the terms heroin, opiate, methadone, substance abuse, fetal development, pregnancy complications, neonatal mortality and abnormalities, infant-newborn, and all subheadings. Review articles and reference lists from identified articles were also reviewed.

\section{Study selection}

Studies were selected if they provided sufficient data for comparisons to be made between neonatal mortality in infants of women who used opiates and those who did not.

\section{Data extraction}

Data were extracted on type of maternal addiction, mean number of antenatal visits, neonatal mortality, and major attributed causes of neonatal mortality.

\section{Main results}

7 studies met the selection criteria. The association between neonatal mortality and use of opiates were evaluated in 3 groups of women: (a) women who continued to use illicit heroin throughout pregnancy; (b) women stabilised on methadone at the time of conception or shortly after; and (c) women who used heroin well into pregnancy with late entry into methadone treatment or who continued to use illicit heroin during pregnancy while receiving methadone. The table shows pooled estimates of the relative risk (RR) for neonatal mortality for maternal heroin use, methadone use, heroin and methadone use, any methadone (with or without heroin use), and any opiates.

\section{Conclusion}

The risk of neonatal mortality is increased in pregnant women who continue to use heroin while receiving methadone.

Pooled relative risks (RRs) for neonatal mortality associated with various type of maternal opiate use

\begin{tabular}{ll}
\hline Major type of opiate used during pregnancy & Pooled RR (95\% CI) \\
\hline Heroin use & $3.27(0.95 \text { to } 9.60)^{*}$ \\
Methadone & $1.75(0.60 \text { to } 2.33)^{*}$ \\
Heroin and methadone & $6.37(2.57$ to 14.68$)$ \\
Any methadone (with or without heroin use) & $3.00(1.50$ to 5.88$)$ \\
Any opiate & $2.55(1.89$ to 3.38$)$ \\
\hline
\end{tabular}

*Not significant

Source of funding: not stated.

For correspondence:Dr G Hulse, Department of Psychiatry and Behavioural Science, Queen Elizabeth Medical Centre, Nedlands, Western Australia 6009, Australia. Fax +61 893463828.

\section{Commentary}

This meta-analysis by Hulse et al of articles published over a 30 year time span is welcome and timely. With the steeply incremental increase in patients dependent on opiates presenting to drug services, the numbers of pregnant women who are dependent on opiates has also increased markedly. However, individual studies reporting on the association between opiate use in pregnancy and neonatal mortality have tended to provide conflicting information.

Hulse et al examined only those studies with controlled populations in 3 groups of women using opiates; firstly, women who continued to use illicit heroin throughout pregnancy; secondly, women stabilised on methadone; and thirdly, women who entered methadone treat- ment in the latter stages of pregnancy. They found that there were sufficient studies to make comparisons between neonatal mortality in these 3 groups of women using opiates and the control groups who were not opiate users.

Although the authors noted that none of the 7 studies in the meta-analysis controlled for any confounding effects of lifestyle, they concluded that women using heroin well into pregnancy with late induction onto methadone treatment have a higher risk of neonatal mortality than those using heroin alone or those who are inducted onto methadone early in pregnancy.

Although lifestyle factors have, in all probability, a bearing on these findings, the information from this review does have implications for clinical practice. In particular, pregnant women should be actively encouraged to enter methadone treatment early in pregnancy and to receive active antenatal care and advice about how their lifestyle may influence the health of the fetus. Furthermore, the authors note that different opiates in combination with poor antenatal care and low birth weight of the neonate have differing associations with neonatal mortality. They urge that well designed studies on such associations are conducted. Of particular concern are the effects of heroin use in pregnancy before and after methadone treatment has started.

Judith Myles, MB, BCH, MRCPsych Blackberry Hill Hospital Bristol, UK 